\title{
Infections bactériennes chez la souris mucoviscidosique: un modèle de la maladie pulmonaire humaine
}

Bien que $95 \%$ de la morbidité et de la mortalité dans la mucoviscidose soient dus à l'atteinte pulmonaire, les relations entre l'anomalie du gène en cause dans la maladie, le CFTR, et les lésions pulmonaires observées ne sont pas connues. Ce que l'on constate, c'est que la maladie pulmonaire est en règle absente chez le nouveauné alors que chez les patients plus âgés on retrouve la coexistence d'un mucus hypervisqueux et d'infections pulmonaires opportunistes qui entraînent la destruction du poumon dans un processus inflammatoire avec perte progressive de la fonction pulmonaire. L'absence de l'hydratation du mucus est vraisemblablement due, au moins en partie, à la diminution des mouvements de chlore à travers les cellules épithéliales. Mais le dysfonctionnement du CFTR conduit-il directement à l'accumulation d'un mucus visqueux qui favorise l'infection, ou existe-t-il une réponse anormale aux agents pathogènes caractéristiques de la mucoviscidose, responsable de la production excessive de sécrétions visqueuses [1] ? Un des moyens de répondre à de telles questions est d'étudier un modèle animal de mucoviscidose. Quatre modèles murins créés par la technique d'inactivation spécifique de gène dans les cellules souches embryonnaires ont été jusqu'à présent rapportés [2]. Les groupes de recherche concernés, localisés en Caroline du Nord (USA), Cambridge (Grande-Bretagne), Houston (TX, USA) et Édimbourg (Grande- Bretagne), ont utilisé diffé1046 l'homologue murin du gène $C F / R$ $\left(\mathrm{m} / \mathrm{s} n^{\circ} 2\right.$, vol. 8 , p. $189 ; n^{\circ} 7$, vol. 8, p. 653 et $n^{\circ} 8$, vol. 8, p. 879). Les trois premiers groupes ont créé des allèles nuls (mutation par remplacement) sans aucune expression résiduelle de CFTR. Le résultat est l'obtention de souris mutantes homozygotes possédant un phénotype extrêmement sévère avec la majorité des animaux (> $80 \%$ ) décédant dans les premiers jours de vie d'obstruction intestinale complète. A l'inverse, les souris créées par le groupe de D. Porteous à Édimbourg ont un phénotype peu sévère. Ils ont pour cela produit une mutation insertionnelle au niveau de l'exon 10 ( $\left.c f t^{m I I C L U}\right)$ qui permet d'obtenir un bas niveau résiduel d'expression de $C F^{2} T R$ par épissage alternatif. Bien que ces souris présentent des anomalies électrophysiologiques typiques de mucoviscidose, l'incidence de décès néonatal par obstruction intestinale est de $5 \%$ à $10 \%$, ce qui correspond à l'incidence de l'iléus méconial chez les patients mucoviscidosiques. Aucune des souris rapportées jusqu'à ce jour ne représente le modèle idéal de mucoviscidose. Une des approches suivie pour améliorer les modèles sans aucune expression résiduelle de $C F^{2} T R$ est de corriger le défaut intestinal létal par expression ciblée du CFTR humain à l'aide d'un promoteur spécifique de l'intestin $\left(\mathrm{m} / \mathrm{s} n^{\circ} 3\right.$, vol. 11 , p. 492). Des efforts sont actuellement réalisés pour produire des souris avec un gène $C I F R$ présentant les mêmes défauts moléculaires que chez l'homme avec notamment la délétion de la phénylalanine ( $\Delta \mathrm{F} 508)$ qui est conser- vée chez la souris. Mais la survie prolongée du modèle de l'équipe d'Edimbourg offre d'importants avantages. Ce groupe vient de rapporter l'effet d'infections bactériennes pulmonaires chez les souris

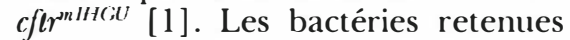
ont été Staphylococcus aureus qui est le colonisateur pulmonaire le plus fréquent et Burkholderia cepacia qui est rarement présent dans les stades précoces de la maladie pulmonaire mais qui entraîne, chez certains malades, une pneumonie fulminante. Après administration par nébulisation des souches bactériennes (cultivées à partir d'isolats de patients mucoviscidosiques) chez des souris cftrmllici/ cftr ${ }^{n ! H(i l)}$ et des souris normales, les auteurs ont montré que, malgré une considérable variation entre les animaux, les souris mutantes retenaient plus, et de manière significative, $S$. aureus ( $\mathrm{p}<0,002)$ et B. cepacia $(p=0,05)$ que les souris normales. En outre, les souris $c f t r^{m I I I C U} / c f t r^{m I H G U}$ montrent une incidence accrue de bronchiolite, de rétention de mucus et d'inflammation en réponse à $S$. aureus et de pneumonie après administration de $B$. cepacia. Ces lésions sont plus diffuses que chez les souris normales. Les résultats suggèrent, en outre, que le dysfonctionnement du CFrR ne conduit pas directement à une rétention excessive de mucus mais plutôt que celle-ci est secondaire à l'infection microbienne. Ainsi donc, la maladie pulmonaire en réponse à l'infection pulmonaire des souris du groupe d'Édimbourg est un modèle qui va permettre de disséquer 
la pathogénie de la maladie pulmonaire mucoviscidosique et d'évaluer l'efficacité d'agents thérapeutiques, pharmacologiques et génétiques. Toutefois, il reste un défi majeur: étudier l'infection des souris

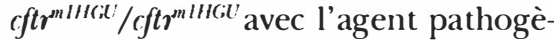
ne le plus délétère dans la mucoviscidose, Pseudomonas aeruginosa. P. aeruginosa n'est pas un colonisateur pulmonaire précoce mais va infecter plus de $90 \%$ des patients [3]. La difficulté vient du fait qu'il existe plusieurs sous-types de bactéries et que, durant l'infection humaine, il y a transformation de souches non mucoïdes en des souches mucoïdes [2]. Les modèles murins de mucoviscidose ont incontestablement beaucoup à nous apprendre sur la maladie humaine.

E.D.

1. Davidson DJ, Dorin JR, Mclachlan (;, Ranaldi V, L amb I), Doherty C, Govan J, Porteous I). L ung disease in the cystic fibrosis mouse exposed 10 bacterial pathogens. Nature Genet 1995; 9 : $351-7$. 2. Anonyme. Symptoms in a "leaky" mouse Vature (ienet 1995; 9: 331-2.

3. Pennacino-Sauvage M, Hulen C. Implantation "t persistance des souches mucoides de Pseudomonas aeruginosa dans les poumons de malades atteints de mucoviscidose. médecine/sciences 1990 (j) : 886-94.

\section{BRÈVES}

Des souris déficientes en récepteur de la dioxine. Le récepteur de la dioxine et d'autres xénobiotiques appartient à la famille des récepteurs nucléaires et se fixe à des séquences spécifiques d'ADN, notamment en amont des gènes codant pour les cytochromes p450 [1]. Fernandez-Salguero et al., de l'équipe de Franck Gonzalez (NIH, Bethesda, CA, USA) [1] rapportent dans Science les résultats de l'invalidation par recombinaison homologue du gène codant pour ce récepteur [2]. Deux anomalies dominent chez les homozygotes: une altération hépatique caractérisée par une fibrose importante des espaces portes avec infiltration inflammatoire des canaux biliaires et altérations hépatocytaires, probablement secondaires; et une déplétion en lymphocytes périphériques dans la rate et les ganglions lymphatiques, sans anomalie de développement thymique. Les animaux meurent probablement d'une combinaison des altérations hépatiques et du déficit immunitaire. Les lésions hépatiques pourraient être dues à l'absence de détoxification de toxines endogènes ou exogènes par le foie mais les causes des troubles immunitaires restent tout à fait obscures. Cependant, ce modèle devrait permettre de mieux comprendre le rôle du récepteur de la dioxine, en dehors de sa fonction de détoxification; si on parvient à prolonger la survie de ces animaux, il pourait être également possible d'étudier ceux qui, dans les effets de la dioxine, passent par la liaison aux récepteurs et ceux ayant d'autres mécanismes.

[1. Beaune, P. médecine/sciences $1986 ; 2$ : 358-63.]

[2. Fernandez-Salguero P. Science 1995 ; 268 : 722-6.]
L'image interne en trois dimensions. Les idiotopes sont des déterminants antigéniques portés par un anticorps ou un groupe d'anticorps. L'ensemble des idiotopes d'un anticorps constitue son idiotype $\left(m / s n^{\circ} 6\right.$, vol. 4, p. 382). Les anticorps anti-idiotypiques peuvent parfois entrer en compétition avec des antigènes du monde extérieur pour la fixation d'un anticorps sur les sites spécifiques de reconnaissance. Il a été proposé que les anticorps anti-idiotypiques mimeraient la structure de ces antigènes en exposant des déterminants appelés "images internes" des antigènes externes. Cette théorie est confortée par la mise en évidence d'un mimétisme fonctionnel entre des ligands de récepteurs et des anticorps anti-idiotypiques de ces ligands. L'étude de la structure cristalline d'un complexe idiotope/anti-idiotope (fragment Fv d'un anticorps antilysozyme lié à un anticorps anti-antilysozyme) vient maintenant soutenir cette hypothèse si souvent malmenée [1]. L'anticorps antilysozyme entre en contact avec son autigène et avec l'anticorps anti-idiotopique de manière très similaire. Il semble donc que l'anticorps anti-idiotypique mime le lysozyme dans son interaction avec l'anticorps primaire. Le mimétisme structural entre anti-antilysozyme et lysozyme est confirmé au niveau fonctionnel car l'anticorps antiidiotypique est immunogène et induit une réponse antilysozyme lorsqu'il est injecté à des souris.

[1. Fields BA, et al. Nature 1995 ; $374: 739-42$.
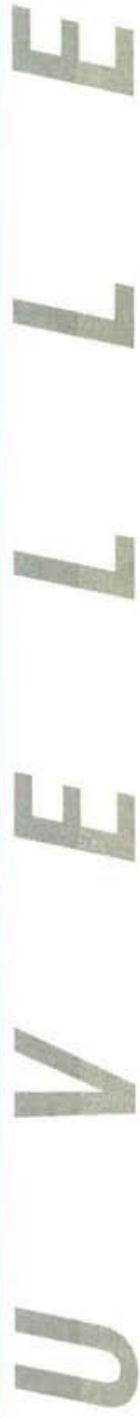